Discrete Comput Geom 37:341-349 (2007)

DOI: $10.1007 / \mathrm{s} 00454-006-1291-6$

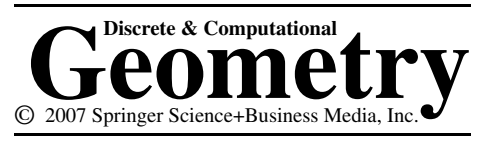

\title{
The Katchalski-Lewis Transversal Problem in $\mathbb{R}^{n}$
}

\author{
Andreas Holmsen* \\ Department of Mathematics, University of Bergen, \\ Johannes Brunsgt. 12, 5008 Bergen, Norway \\ andreash@mi.uib.no
}

\begin{abstract}
Let $F$ be a family of disjoint translates of a compact convex set in the plane. In 1980 Katchalski and Lewis showed that there exists a constant $k$, independent of $F$, such that if each three members of $F$ are met by a line, then a "large" subfamily $G \subset F$, with $|F \backslash G| \leq k$, is met by a line. In this paper we obtain a higher-dimensional analogue containing the Katchalski-Lewis result. Also we give two constructions of families of pairwise disjoint translates of the unit ball in $\mathbb{R}^{3}$ which answer some related questions.
\end{abstract}

\section{Introduction}

Let $F$ be a family of convex sets in $\mathbb{R}^{n}$. A line meeting each member of $F$ is called a line transversal. In general a $k$-flat $(0 \leq k<n)$ meeting every member of $F$ is called a $k$-transversal, and for $k=n-1$ we also say hyperplane transversal. When $F$ has a $k$-transversal we say that $F$ has the $T_{k}$ property. If every $m$ or fewer members of $F$ admit a $k$-transversal we say that $F$ has the $T_{k}(m)$ property.

Much effort has been devoted to finding conditions that guarantee a transversal to a family of convex sets. For the most general families necessary and sufficient conditions for a hyperplane transversal were discovered in a sequence of papers: [1], [6], [8], [15], and [17]. Common for all of these results are that they require a global ordering condition. If one wishes to obtain results without such an ordering condition it is necessary to restrict the families of convex sets in a suitable way.

A special type of families that has been studied in recent decades is families of translates of a compact convex set. In 1989 Tverberg [18] proved a long-standing conjecture by Grünbaum [7] which states that if $F$ is a family of pairwise disjoint translates in the plane, then $T_{1}(5) \Rightarrow T_{1}$. (Such a result is often called a Helly-type theorem).

In 1980 Katchalski and Lewis [16] considered what happens if the $T_{1}(5)$ property is weakened and only the $T_{1}(3)$ property is assumed. For families of disjoint translates

\footnotetext{
* Supported by the Research Council of Norway.
} 
in the plane which have the $T_{1}(3)$ property they found that there is a line that meets "almost" all the translates.

To be more specific, we say that $F$ has the $T_{k}-c$ property if there exists a subfamily $G \subset F$ with $|F \backslash G| \leq c$ such that $G$ has the $T_{k}$ property. The Katchalski-Lewis theorem states that there exists a constant $c$ such that if $F$ is a family of pairwise disjoint translates in the plane, then $T_{1}(3) \Rightarrow T_{1}-c$.

The problem of determining the best possible constant $c$ has been studied by various authors in [9], [10], [14], and [19].

In the present paper we give an extension of the Katchalski-Lewis theorem to $\mathbb{R}^{n}$. Our first result is the following.

Theorem 1. For every $n \geq 2$ there exists a minimal non-negative integer $c(n)$ such that for any family of disjoint translates in $\mathbb{R}^{n}$, we have

$$
T_{1}(3) \Rightarrow T_{n-1}-c(n) \text {. }
$$

Theorem 1 follows as a consequence of

Theorem 2. For every $k \geq 0$ there exists a minimal positive integer $d(k)$ such that for any family of translates in $\mathbb{R}^{2}$, of depth $k$, we have

$$
T_{1}(3) \Rightarrow T_{1}-d(k)
$$

(The depth of a finite family of sets in the plane is defined as the maximum number of sets from the family covering a single point.)

In particular it is shown in Section 2 that given a family $F$ of pairwise disjoint translates in $\mathbb{R}^{n}$, we can always find an appropriate 2 -flat, $P$, such that the orthogonal projection of $F$ into $P$ is a family of translates of depth at most $k=k(n)$. Since the $T_{1}(3)$ property is preserved under projections, by Theorem 2 , there is a line in $P$ that meets all but at most $d(k)$ members of the projected family. This line lifts to a hyperplane that meets all but at most $d(k)$ members of $F$, so the result follows.

Sections 3 and 4 are devoted to constructions of families of translates of the Euclidean unit ball in $\mathbb{R}^{3}$. Recently such families have received some attention. First it was shown in [11] that there is a Helly-type theorem for line transversals. Improvements on this result were made in [5], and finally in [4] it was shown that for families of disjoint translates of the Euclidean unit ball in $\mathbb{R}^{n}$ we have

$$
T_{1}(4 n-1) \Rightarrow T_{1} \text {. }
$$

In contrast to this, it was shown in [12] that given positive integers $k$ and $n$ there exists a convex polytope $C=C(k, n) \subset \mathbb{R}^{3}$ and a family of pairwise disjoint translates of $C$ which has the $T_{1}(n)$ property but not the $T_{1}-k$ property.

In Section 3 we describe a family of $6 n(n>0)$ disjoint unit balls in $\mathbb{R}^{3}$ with the $T_{1}(3)$ property, such that any line meets at most $4 n$ of the balls. This construction answers a question raised by Kaiser [14].

In Section 4 we describe a family of $2 n+1(n>1), 1$-separated, unit balls in $\mathbb{R}^{3}$ with the $T_{2}(2 n)$ property, but with no plane meeting them all. This construction answers a question raised by Aronov et al. [3]. 


\section{Proof of Theorem 1}

First we note that Theorem 2 follows easily from the original Katchalski and Lewis paper: Corollary 3 of [16].

We express a family of translates as $F=\left\{K+v_{i}, i \in I\right\}$ where $K \subset \mathbb{R}^{n}$ is a compact convex set and the $v_{i} \in \mathbb{R}^{n}$ are the translation vectors. The following reductions will be useful.

We may assume that $K$ is centrally symmetric with interior points. First, assume $\operatorname{dim}(K)<n$. If some pair of translates, $X$ and $Y$, are contained in a common hyperplane, $H$, then every line transversal to $X$ and $Y$ is contained in $H$, and thus, by the $T_{1}(3)$ property, all the translates of $F$ are contained in $H$ and we are done. Alternatively, the members of $F$ lie in distinct parallel hyperplanes, inducing an ordering on $F$, such that each triple of $F$ is intersected by a line respecting the given ordering (Hadwiger's condition), and by a theorem of Katchalski [15], $F$ has $T_{n-1}$, and again there is nothing to prove. Therefore we can assume $\operatorname{dim}(K)=n$, that is, $K$ has interior points.

Next, let $K_{\text {sym }}=\frac{1}{2} K-\frac{1}{2} K$. The following facts are easily verified: $\left(K+v_{i}\right) \cap(K+$ $\left.v_{j}\right)=\emptyset$ if and only if $\left(K_{\mathrm{sym}}+v_{i}\right) \cap\left(K_{\mathrm{sym}}+v_{j}\right)=\emptyset$, and $\left\{K+v_{i} ; i \in I\right\}$ has the $T_{n-1}$ property if and only if $\left\{K_{\text {sym }}+v_{i} ; i \in I\right\}$ has the $T_{n-1}$ property. Now suppose $K+v_{1}$, $K+v_{2}, K+v_{3}$ admit a line transversal in the stated order, and let $\pi$ be the orthogonal projection in the direction of the line spanned by $v_{1}$ and $v_{3}$. Then $\pi\left(K+v_{1}\right)=\pi\left(K+v_{3}\right)$ and $\pi\left(K+v_{2}\right) \cap \pi\left(K+v_{1}\right) \neq \emptyset$. By linearity of $\pi$ we have $\pi\left(K_{\text {sym }}\right)=[\pi(K)]_{\text {sym }}$, and therefore $K+v_{1}, K+v_{2}, K+v_{3}$ admit a line transversal if and only if $K_{\text {sym }}+v_{1}$, $K_{\text {sym }}+v_{2}, K_{\text {sym }}+v_{3}$ admit a line transversal. In other words $\left\{K+v_{i} ; i \in I\right\}$ has the $T_{1}$ (3) property if and only if $\left\{K_{\mathrm{sym}}+v_{i} ; i \in I\right\}$ has the $T_{1}(3)$ property. This implies that Theorem 1 is true for $\left\{K+v_{i} ; i \in I\right\}$ if and only if it is true for $\left\{K_{\text {sym }}+v_{i} ; i \in I\right\}$. Therefore it suffices to prove Theorem 1 for $K$ centrally symmetric.

We may assume that $K$ has diameter 1 and volume at least $1 / n !$. This can be arranged as follows. Let $A$ be an affine transformation of $\mathbb{R}^{n}$ which maps $K$ to $A(K)$ of diameter 1 and volume at least $1 / n$ !. The existence of $A$ follows easily from John's theorem [13] (or rather its dual). Clearly Theorem 1 is true for $A(F)$ if and only if is true for $F$. This justifies our claim.

Lemma 3. There exists a cylinder of radius $\frac{3}{2}$ that contains every member of $F$.

Proof. We prove the lemma first for the case when $K$ is the Euclidean ball of diameter 1. Let $X$ and $Y$ be the pair with maximum distance between the centers, and let the line through the centers be the $x_{1}$-axis. Let $C$ be the cylinder of radius $\frac{3}{2}$ with the $x_{1}$-axis as its central line. We claim that each ball of the family is contained in $C$. Assume the contrary, and let $Z$ be a ball that is not contained in $C$, and let $x, y, z$ be the centers of $X$, $Y, Z$, respectively. For any triple of points $p, q, r$, let $l_{p q}$ denote the distance between $p$ and $q$ and let $h_{p q}(r)$ denote the distance from $r$ to the line containing $p$ and $q$. We have

$$
l_{x y} h_{x y}(z)=l_{x z} h_{x z}(y)=l_{y z} h_{y z}(x)
$$

and by the choice of $x$ and $y$ it follows that $h_{x y} \leq h_{x z}, h_{y z}$. Now it is easily verified that $Z$ is not contained in $C$ if and only if $h_{x y}>1$. However, then $h_{x z}$ and $h_{y z}$ are also greater 
than 1 , and it is easily seen that this implies that $X, Y, Z$ cannot have a line transversal, which contradicts the $T_{1}(3)$ property. (Note that disjointness was not required for this argument.)

Now for the general case where $F$ is a family of disjoint translates of the oval $K$. Since $K$ is centrally symmetric of diameter 1 it can be inscribed in an $n$-ball $B^{n}(K)$ of diameter 1 . Obviously, the family $B^{n}(F)=\left\{B^{n}(K)+v_{i}, i \in I\right\}$ inherits the property $T_{1}(3)$ from $F$ and we can apply the above argument to $B^{n}(F)$ obtaining a cylinder $C$ of radius $\frac{3}{2}$ which contains each member of $B^{n}(F)$, thus also containing each member of $F$.

The direction of the cylinder $C$ is called the extremal direction of $F$. (Note that the extremal direction need not be unique).

Lemma 4. Let $F$ be a family of disjoint translates in $\mathbb{R}^{n}(n \geq 3)$ with the $T_{1}(3)$ property, and let $P$ be a 2-flat containing the extremal direction of $F$. Then the orthogonal projection of $F$ into $P, P(F)$, is a family of translates, with the $T_{1}(3)$ property, of depth at most $4 \cdot n ! \cdot 3^{n-2}$.

Proof. The fact that the projected family has the $T_{1}(3)$ property is trivial. We may assume that the $x_{1}$-axis is the extremal direction of $F$. Let $P$ be spanned by the $x_{1}$-and $x_{2}$-axes. Let $x$ be a point in $P$. If $x \in P(K)$ then $P(K)$ (i.e. the orthogonal projection of $K$ into $P$ ) is contained in a square region of sidelength 2 centered at $x$ with sides parallel to the $x_{1}$ - and $x_{2}$-axis. By Lemma $3, K$ is contained in a box of volume $4 \cdot 3^{n-2}$. Since the members of $F$ are pairwise disjoint and $K$ has volume at least $1 / n !$ the result follows.

Theorem 1 now follows from Theorem 2 and Lemma 4.

\section{A Question by Kaiser}

In [14], Kaiser raises the following question: Is there a constant $c$ such that for any finite family of disjoint unit balls in $\mathbf{R}^{3}$ in which each three members have a line transversal, there is a line intersecting all except at most $c$ of the balls?

Here we give a negative answer to this question:

Proposition 5. There exists a family of $6 n$ pairwise disjoint unit balls in $\mathbb{R}^{3}$ that has $T_{1}(3)$, but where any line meets at most $4 n$ of the balls.

Proof. Let $n \geq 1$ and $F=A \cup B \cup C \cup A^{\prime} \cup B^{\prime} \cup C^{\prime}$ where

$$
\begin{array}{ll}
A=\left\{A_{1}, A_{2}, \ldots, A_{n}\right\}, & A^{\prime}=\left\{A_{1}^{\prime}, A_{2}^{\prime}, \ldots, A_{n}^{\prime}\right\}, \\
B=\left\{B_{1}, B_{2}, \ldots, B_{n}\right\}, & B^{\prime}=\left\{B_{1}^{\prime}, B_{2}^{\prime}, \ldots, B_{n}^{\prime}\right\}, \\
C=\left\{C_{1}, C_{2}, \ldots, C_{n}\right\}, & C^{\prime}=\left\{C_{1}^{\prime}, C_{2}^{\prime}, \ldots, C_{n}^{\prime}\right\},
\end{array}
$$




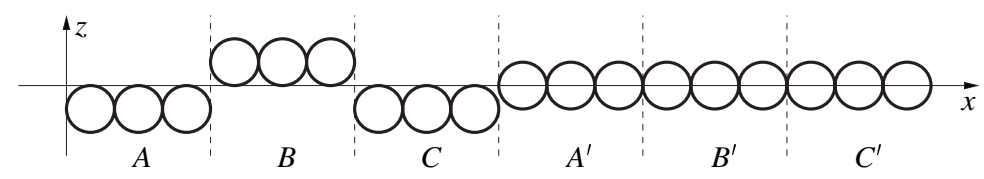

Fig. 1. The case $n=3$. Projection into the $x z$-plane.

are distinct unit balls. The centers of $A_{k}, B_{k}, C_{k}, A_{k}^{\prime}, B_{k}^{\prime}, C_{k}^{\prime}$ are denoted as $a_{k}, b_{k}, c_{k}$, $a_{k}^{\prime}, b_{k}^{\prime}, c_{k}^{\prime}$, respectively, and are given as

$$
\begin{array}{ll}
a_{k}=(2 k-1,0,-1), & a_{k}^{\prime}=(6 n+2 k-1,1,0), \\
b_{k}=(2 n+2 k-1,0,1), & b_{k}^{\prime}=(8 n+2 k-1,-1,0), \\
c_{k}=(4 n+2 k-1,0,-1), & c_{k}^{\prime}=(10 n+2 k-1,1,0)
\end{array}
$$

for $1 \leq k \leq n$.

Different projections of $F$ are illustrated in Figs. 1 and 2. Note that the triples of the form $\left\{A_{i}, B_{j}, C_{k}\right\}$ and $\left\{A_{i}^{\prime}, B_{j}^{\prime}, C_{k}^{\prime}\right\}$ have the $x$-axis as a unique transversal.

We now show that the triples not of the form $\left\{A_{i}, B_{j}, C_{k}\right\}$ and $\left\{A_{i}^{\prime}, B_{j}^{\prime}, C_{k}^{\prime}\right\}$ have transversals different from the $x$-axis. Some of the triples are easy to check; for instance triples of the form $\left\{A_{i}, C_{j}, B_{k}^{\prime}\right\}$. Triples of the form $\left\{A_{i}, B_{j}, C_{k}^{\prime}\right\}$ require a closer look. In fact, by the symmetrical structure of $F$ it suffices to show that a triple $\left\{A_{i}, B_{j}, C_{k}^{\prime}\right\}$ has a transversal different from the $x$-axis. For some $0<\varepsilon<1$, consider the plane $H$ given by $y=\varepsilon$. The intersection of $H$ and the triple $\left\{A_{i}, B_{j}, C_{k}^{\prime}\right\}$ is a family of discs of different radii. In particular, we find that the radius of $A_{i} \cap H$ and $B_{j} \cap H$ is $\sqrt{1-\varepsilon^{2}}$. The radius of $C_{k}^{\prime} \cap H$ is $\sqrt{2 \varepsilon-\varepsilon^{2}}$.

The reader can verify the following: There is a line in the plane $H$ of slope $\varepsilon^{2}$ that will intersect $A_{i} \cap H$ and $B_{j} \cap H$ in interior points. Furthermore, if we let $\varepsilon<$ $1 / 7 n$ we are also guaranteed that the same line will intersect $C_{k}^{\prime} \cap H$ in interior points. Thus the triples of the form $\left\{A_{i}, B_{j}, C_{k}\right\}$ and $\left\{A_{i}^{\prime}, B_{j}^{\prime}, C_{k}^{\prime}\right\}$ have the $x$-axis as a unique transversal, while all other triples have transversals that intersect the members in interior points.

The fact that these transversals intersect the balls in interior points lets us move them slightly in the $x$-direction such that we get disjointness, while preserving the nonhorizontal transversals. We may also translate $A^{\prime} \cup B^{\prime} \cup C^{\prime}$ slightly in the $z$-direction

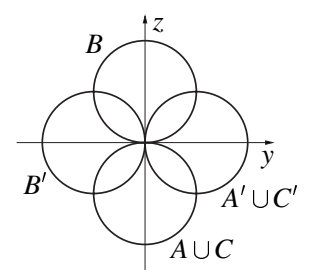

Fig. 2. Projection into the $y z$-plane. 


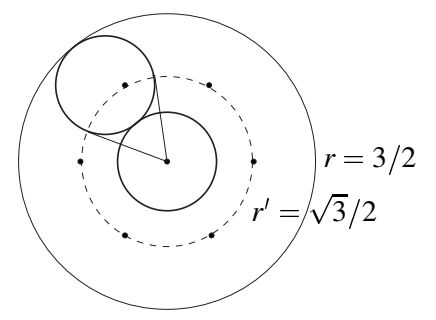

Fig. 3. The seven points sufficient to catch each disc in the projection.

such that they do not meet the $x$-axis, but still have a unique transversal parallel to the $x$-axis. Thus, by the construction, we have the following:

1. $F$ has the $T_{1}(3)$ property.

2. The $x$-axis is a unique transversal for $A \cup B \cup C$ and misses every member of $A^{\prime} \cup B^{\prime} \cup C^{\prime}$.

3. $A^{\prime} \cup B^{\prime} \cup C^{\prime}$ has a unique transversal parallel to the $x$-axis, lying slightly above, which misses every member of $A \cup C$.

4. Any other line (than the two specified above) misses every member of either $A$, $B$, or $C$, and every member of either $A^{\prime}, B^{\prime}$, or $C^{\prime}$.

Therefore any line misses at least $2 n$ members of $F$.

Thus we have given a negative answer to Kaiser's question. Still, one could ask, is the property $T_{1}(3)$ strong enough to guarantee a fractional result? The following result is due to Aronov [2].

Theorem 6. For any family of unit balls in $\mathbb{R}^{3}$ with the $T_{1}(3)$ property there exists seven parallel lines such that each ball meets at least one of the lines.

Proof. Projecting into a plane orthogonal to the extremal direction we obtain a family of unit discs contained in a disc $D$ of radius $r=\frac{3}{2}$. Let $C$ be the circle of radius $r^{\prime}=\sqrt{3} / 2$ concentric to $D$. A unit disc contained in $D$ which does not contain the center of $D$ will intersect $C$ in a circular arc of angular length of at least $\pi / 3$. Therefore any unit disc that does not contain the center of $D$ will contain one of six points on $C$ forming the vertices of a regular hexagon. Thus we have found seven points that together catch every unit disc in $D$, see Fig. 3 . These points lift to lines whose union meets every member of $F$.

\section{Some Related Questions}

In [3] the authors conjecture a Helly-type theorem for hyperplane transversals to unit balls. Here is a counterexample. 


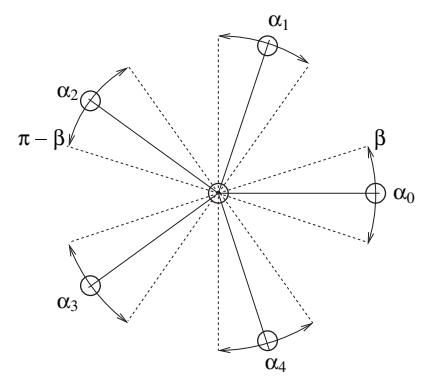

Fig. 4. The case $n=3$. Projection into the $x y$-plane.

Proposition 7. For every $n \geq 2$, there exists a family of $2 n+1$, 1-separated, unit balls in $\mathbb{R}^{3}$ that has $T_{2}(2 n)$, but not $T_{2}$.

(A family of convex sets in $\mathbb{R}^{n}$ is called $k$-separated $(0 \leq k<n)$ if no $k+2$ members admit a $k$-transversal. So 0 -separated is the same disjoint.)

Proof. Let $\alpha_{k}=2 \pi k /(2 n-1)$ for $k=0,1, \ldots, 2 n-2$ and $\varepsilon>0$. Let $A$ and $B$ be unit balls with centers (given in cylindrical coordinates)

$$
a=(0,0,1+\varepsilon), \quad b=(0,0,-(1+\varepsilon))
$$

and let $C_{0}, C_{1}, \ldots, C_{2(n-1)}$ be unit balls with centers

$$
c_{k}=\left(R, \alpha_{k}, 0\right),
$$

where $R$ will be defined later (see Fig. 4). Let $H$ be a common separating tangent plane to $A$ and $B$, and let $n_{H}$ be the normal vector to $H$ with positive $z$-coordinate. The projection of $n_{H}$ into the $x y$-plane makes an angle $\theta_{H} \in[0,2 \pi)$ with the positive $x$-axis. Moreover, the angle $\theta_{H}$ uniquely determines $H$. Thus the set of common separating tangent planes of $A$ and $B$ will be denoted $H(\theta)$, with $\theta \in[0,2 \pi)$.

Consider the ball $C_{0}$. Let the function $D$ be defined as

$$
D(x)=\frac{x+1}{\sqrt{x^{2}+2 x}}, \quad \text { for } \quad x>0 .
$$

It is easily checked that if $R=D(\varepsilon)$ then the plane $H(0)$ tangent to $C_{0}$ from below, and the plane $H(\pi)$ is tangent from above. Now suppose $R>D(\varepsilon)$. Then $H(0)$ and $H(\pi)$ will both miss $C_{0}$, but clearly $H(\pi / 2)$ and $H(3 \pi / 2)$ both meet $C_{0}$. By continuity, there exists a minimal $\beta=\beta(R) \in(0, \pi / 2)$ such that $H(\theta) \cap C_{0} \neq \emptyset$ if and only if $\beta \leq \theta \leq \pi-\beta$ or $\pi+\beta \leq \theta \leq 2 \pi-\beta$. Clearly, $\beta(R)$ is a strictly increasing function.

For $k=0,1, \ldots, 2(n-1)$, let

$$
S_{k}=\left(\alpha_{k}-\beta, \alpha_{k}+\beta\right) \cup\left(-\alpha_{k}-\beta,-\alpha_{k}+\beta\right) .
$$

We choose $R$ sufficiently large such that the union of the $S_{k}$ covers the entire circle, but such that the union of any $2 n-2$ or fewer of the $S_{k}$ does not. Note that this requires us first to choose $\varepsilon$ sufficiently small. 
We now verify the following properties.

1. The family has the $T_{2}(2 n)$ property. The plane $H\left(\alpha_{k}\right)$ will intersect every member except $C_{k}$. The planes $z=\varepsilon$ and $z=-\varepsilon$ will intersect every member except $B$ and $A$, respectively.

2. The family is 1-separated. Clearly when $R$ is sufficiently large, there is no line transversal for triples of the form $A, C_{i}, C_{j}$ or $C_{i}, C_{j}, C_{k}$. If a triple $A, B, C_{i}$ had a line transversal it would have to meet the sets in the order $A B C_{i}$ or $B A C_{i}\left(A C_{i} B\right.$ is excluded since $R$ is large). However, the plane $H\left(\alpha_{i}\right)$ separates $B$ from $A$ and $C_{i}$, thus there can be no line transversal in the order $A B C_{i}$. The plane $H\left(-\alpha_{i}\right)$ excludes the order $B A C_{i}$, so the family is 1-separated.

3. The family does not have the $T_{2}$ property. For a plane to intersect both $A$ and $B$ it must be at least as "steep" as the separating tangents of $A$ and $B$. However, any plane as "steep" or steeper than these, will miss at least one of the $C_{i}$, by construction.

Open Questions. Even though there is no Helly-theorem for plane transversals to unit balls in $\mathbb{R}^{3}$, perhaps there is an analogue to Theorem 1 :

Does there exist a constant $c$ such that for any finite family of disjoint unit balls in $\mathbb{R}^{3}, T_{2}(4) \Rightarrow T_{2}-c$ ?

This would be a special case of a more general conjecture:

Conjecture 8. For all integers $k$ and $d$, where $0<k<d$, there exists a minimal non-negative integer $c(k, d)$ such that for any family $F$ of pairwise disjoint translates of a compact convex set in $\mathbb{R}^{d}$ the following always holds:

$$
T_{k}(k+2) \Rightarrow T_{n-1}-c(k, d) .
$$

Theorem 1 is the case $k=1$ of this conjecture. Note that to prove Conjecture 8 it is sufficient to prove the case $k=d-1$, simply because

$$
T_{k}(k+2) \Rightarrow T_{k+1}(k+3)
$$

If we let $n=2$ in the example of Proposition 7 we have shown that in Conjecture 8 the constant $c(2,3) \geq 1$. We make a point of this here because we have not been able to find an example showing that the constant $c(1,3)>0$. In fact, we conjecture that such an example does not exist.

Conjecture 9. Let $F$ be a family of pairwise disjoint translates of a compact convex set in $\mathbb{R}^{3}$. Then

$$
T_{1}(3) \Rightarrow T_{2}
$$




\section{Acknowledgments}

Special thanks to Boris Aronov for many helpful discussions. Also the construction of Proposition 7 was found independently by B. Aronov, V. Koltun, and M. Sharir.

\section{References}

1. L. Anderson and R. Wenger. Oriented matroids and hyperplane transversals. Adv. Math. 119 (1996), $117-125$.

2. B. Aronov. Personal communication.

3. B. Aronov, J. E. Goodman, R. Pollack, and R. Wenger. On the Helly number for hyperplane transversals to unit balls. Discrete Comput. Geom. 24 (2000), 171-176.

4. O. Cheong, X. Goaoc, A. Holmsen, and S. Petitjean. Helly-type theorems for line transversals to disjoint unit balls in any dimension. In preparation (2005).

5. O. Cheong, X. Goaoc, and H.-S. Na. Geometric permutations of disjoint unit spheres. Submitted for publication in Comput. Geom.

6. J. E. Goodman and R. Pollack. Hadwiger's transversal theorem in higher dimensions. J. Amer. Math. Soc. 1 (1988), 301-309.

7. B. Grünbaum. On common transversals. Arch. Math. 9 (1958), 465-469.

8. H. Hadwiger. Über eibereiche mit gemeinsamer treffgeraden. Port. Math. 16 (1957), 23-29.

9. A. Heppes. On the Katchalski-Lewis transversal conjecture for $T$ (3)-families of congruent discs. To appear in Discrete Comput. Geom..

10. A. Holmsen. New bounds on the Katchalski-Lewis transversal problem. Discrete Comput. Geom. 29 (2003), 395-408.

11. A. Holmsen, M. Katchalski, and T. Lewis. A Helly-type theorem for line transversals to disjoint unit balls. Discrete Comput. Geom. 29 (2003), 595-602.

12. A. Holmsen and J. Matoušek. No Helly theorem for stabbing translates by lines in $\mathbb{R}^{3}$. Discrete Comput. Geom. 31 (2004), 197-206.

13. F. John. Extremum problems with inequalities as subsidiary conditions. Pages 187-204 in Studies and Essays Presented to R. Courant on His 60th Birthday, Interscience, New York, 1948.

14. T. Kaiser. Line transversals to unit discs. Discrete Comput. Geom. 28 (2002), 379-388.

15. M. Katchalski. Thin sets and common transversals. J. Geom. 14, 103-107.

16. M. Katchalski and T. Lewis. Cutting families of convex sets. Proc. Amer. Math. Soc. 79 (1980), 457-461.

17. R. Pollack and R. Wenger. Necessary and sufficient conditions for hyperplane transversals. Combinatorica 10 (1990), 307-311.

18. H. Tverberg. Proof of Grünbaum's conjecture on common transversals for translates. Discrete Comput. Geom. 4 (1989), 191-203.

19. H. Tverberg. On geometric permutations and the Katchalski-Lewis conjecture on partial transversals for translates. Pages 351-361 in Discrete and Computational Geometry (New Brunswick, NJ, 1989/1990), American Mathematical Society, Providence, RI, 1991.

Received November 21, 2003, and in revised form August 23, 2004, and November 3, 2005.

Online publication March 2, 2007. 